
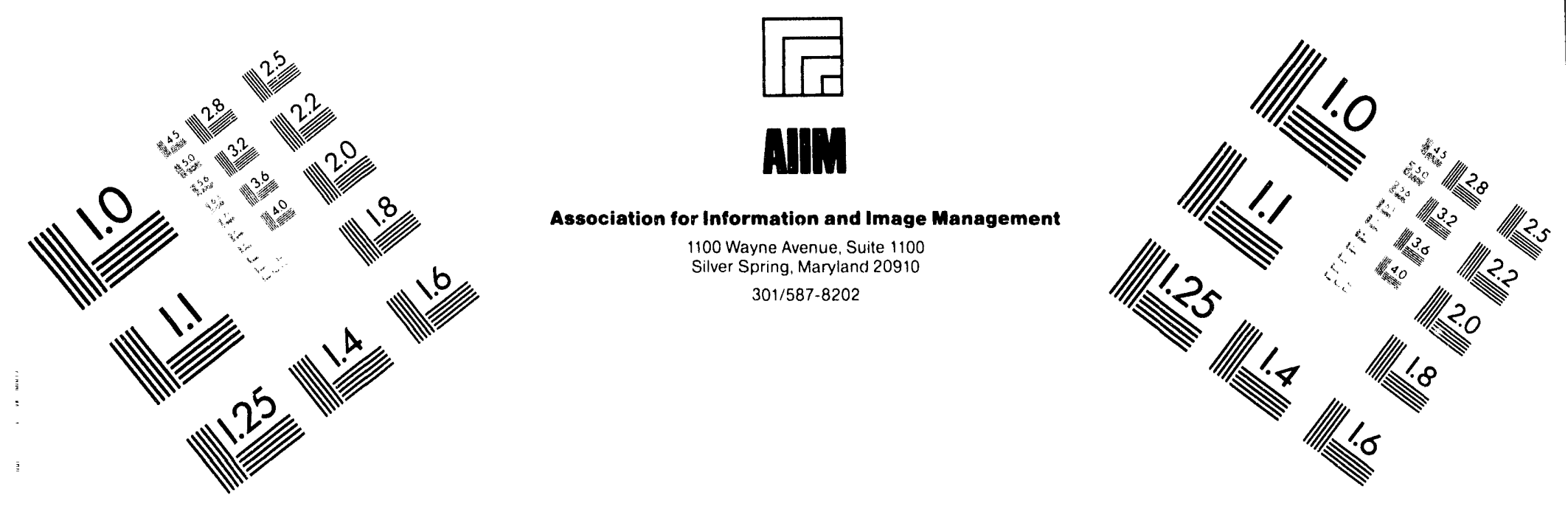

\title{
Centimeter
}

${ }_{1}$

Inches
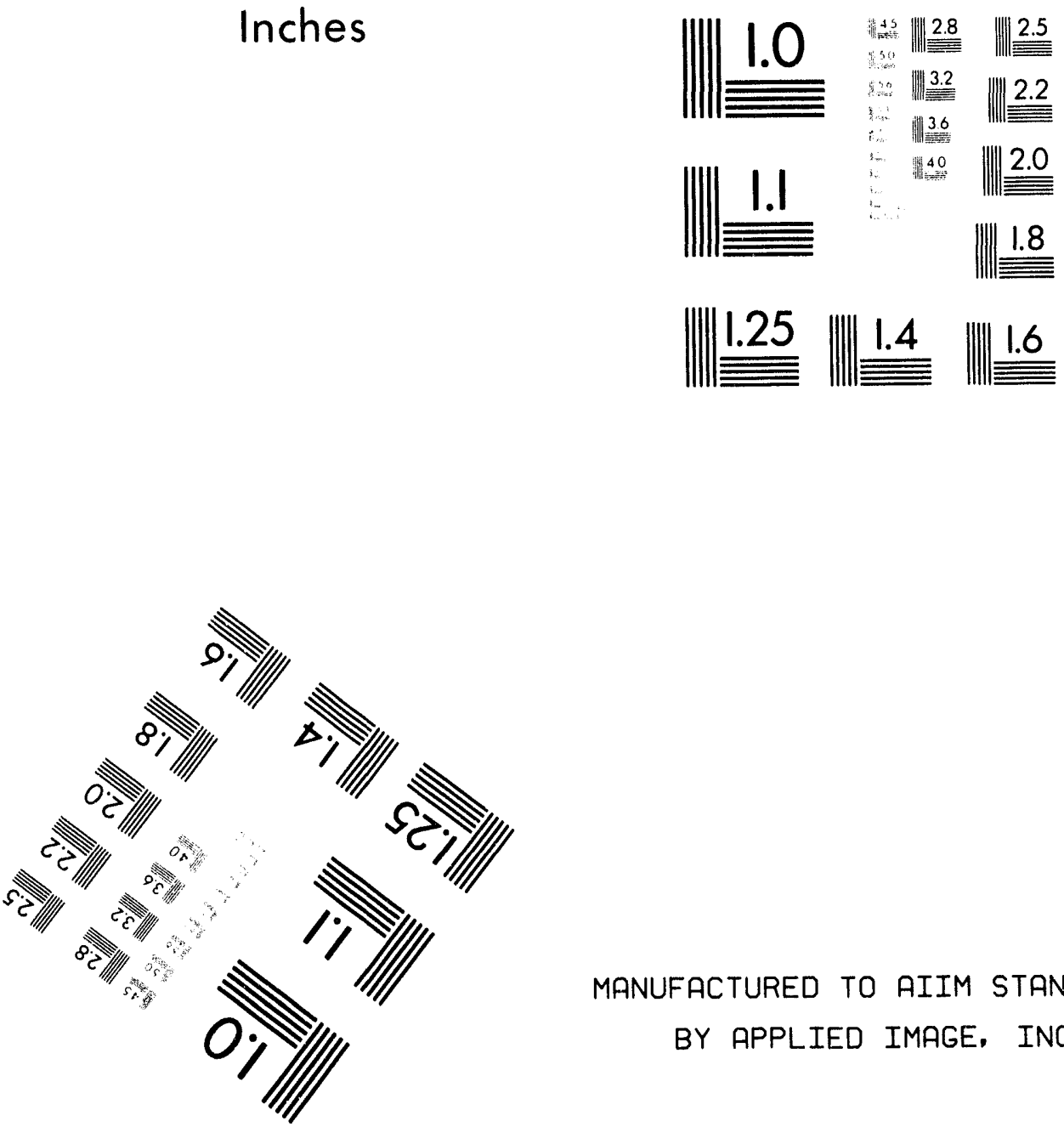

MANUFACTURED TO AIIM STANDARDS

BY APPLIED IMAGE, INC.

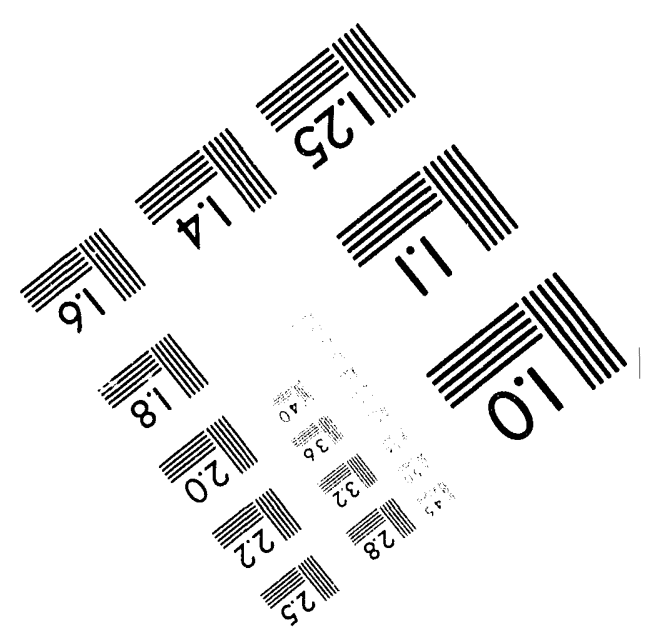


UCRL-CR-115793

\section{Application of Multiquadric Method for Numerical \\ Solution of Elliptic Partial Differential Equations}

\section{Maithili Sharan}

E.J. Kansa

Suman Gupta

January 1994

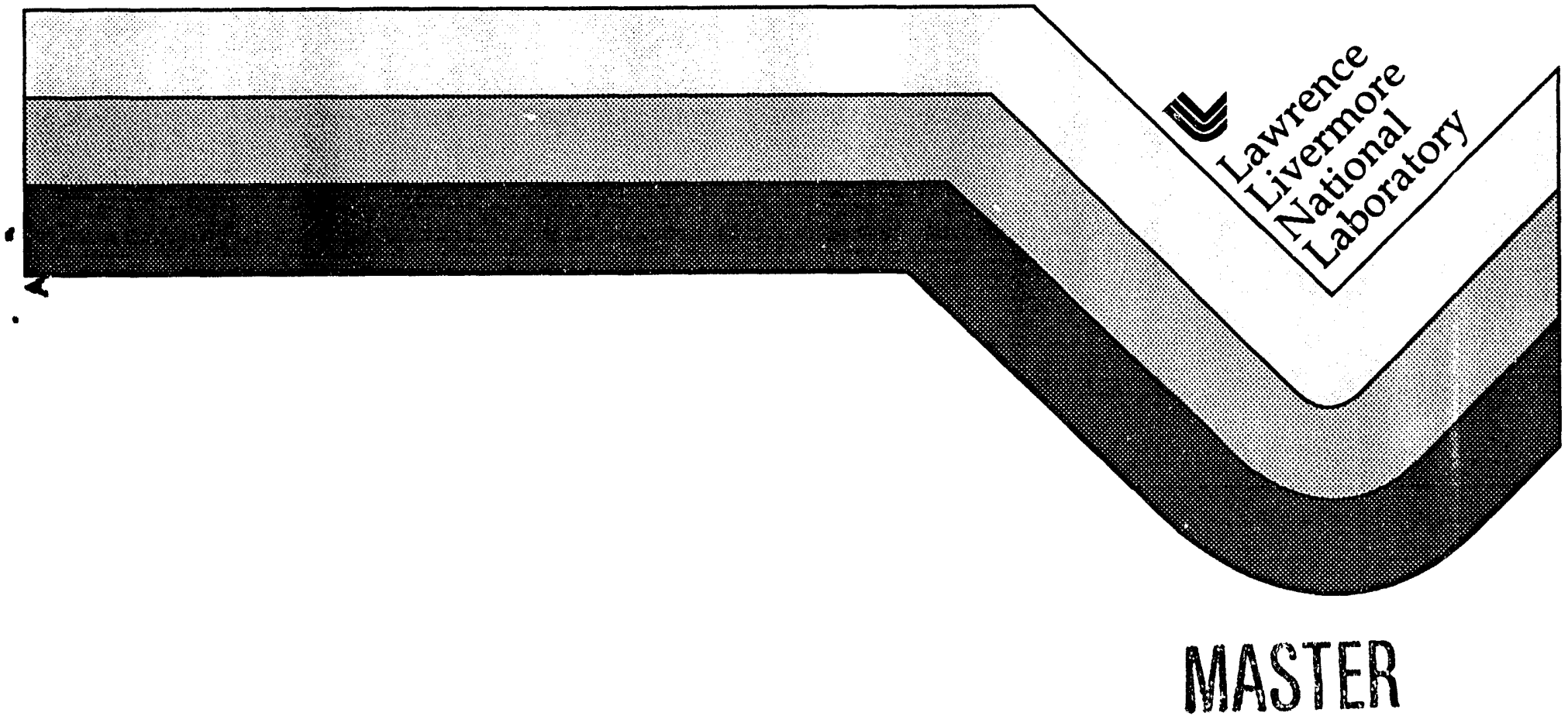




\section{DISCLAIMER}

Work performed under the auspices of the U.S. Department of Energy by Lawrence Livermore National I aboratory under contract number W-7405-E.VG-48.

This document was prepared as an account of work sponsored by an agency of the United States Government. Neither the United States Government nor the University of California nor any of their employees. makes any warranty, express or implied, or assumes any legal liability or responsibility for the accuracy, completeness. or usefulness of any information. apparatus, product, or process disclosed, or represents that its use would not infringe prisately owned rights. Reference herein to any specific commercial products, process, or service by trade name, trademark, manufacturer. or otherwise, does not necessarily constitute or imply its endorsement, recommendation, or favoring by the linited States Government or the Universit! of California. The views and opinions of authors expressed herein do not necessarily state or reflect those of the United States Government or the Unisersity of California, and shall not be used for advertising or product endorsement purposes. 
Appl 1cation of Multiquadric Mothod for Numerical Sol ution of El11pt1c Fartial Difforential Equations.

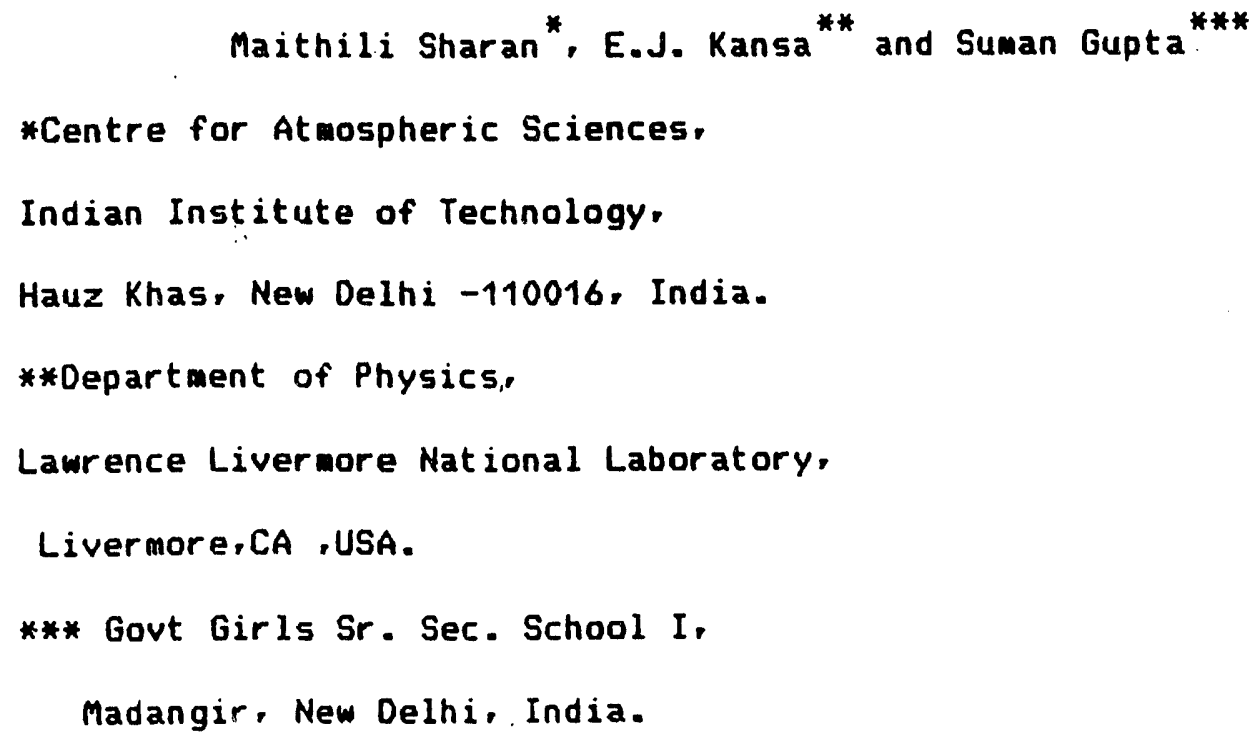


Abstract

We have used the multiquadric (MQ) approximation scheme for the solution of elliptic partial differential equatlons with Dirichlet and/or Neumann boundary conditions. The scheme has the advantage to use tha data points in arbitrary locations with an arbitrary ordering. Two dimensional Laplace, Poisson and Biharmonic equations describing the various physical processes, have been taken as the test examples. The agreement is found to be very good between the computed and exact solutions. The method also provides an execellent approximation with curve boundrary. 


\section{Iritroduct 1or}

In general, mathematical description of physical processes leads to the partial differential equations (PDE). In some cases, an exact solution to PDE can be obtained using analytical tools such as method of reflection and superposition. separation of variables and integral transforms etc. The approximate analytical solution of PDE can be found by using perturbation methods (Nayfeh(1973), Van Dyke (1975), Kevorkian \& Cole (1981)), method of successive approximation (Parlange (1971)) and orthogonal functions (Tsang (1960)).

The solution of equations which more closely model experimental and practical situations is possible by the methods of numerical analysis. The equations can be solved by using finito difference, finite element and boundary element methods. When the boundary conditions and domains are irregular, the finite difference scheme becomes complex. It can be overcome to a greater extent by using the method of finite elements.

It is difficult to use finite difference methods on arbitrarily scattered data points. Hárdy in 1968 (Hardy (1990)) introduced for the first time multiquadric (ne) wethod for the construction of approximate 2-dimensional surfaces through field data. The importance anu usefulness of the ne method is recognised from the fact that it has been applied in geology. geophysics, survey apping, photogrammetry, remote sensing and signal processing, geography and digital terrain models, and hydrology. Hardy (1990) has presented an excellent review summary on the various applications of mo method during last twenty years. Based on the comparison of different scattered data scheves. Franke (1982) has concluded that mo norforme tha hact in 
accuracy and ease of implementation even against various. finlte element schemes. Kansa (1990 a) has explored MQ scattered data scheme in interpolation of data points to approximate surfaces and estimating partial derivatives. Kansa (1990b) has pointed out that the interpolation scheme is intimately connected with the solution of POEs and he applied successfully this novel technique for the solution of POEs in computational fluid dynamics. Dubal et al (1992) and Dubal (1992) have used the mo schene for the solution of a three dimensional elliptic partial differential equation describing the initial state of the collision of two-black holes. Makroglou (1992) has used Ma method to solve Fredholm integral and Integro-differential equations.

The no method replaces the classical finite difference and finite element spatial discretization schemes by a exponentially convergent, grid free scattered data approximation scheme.

Finite difference and finite element methods use low order polynomial basis functions. MQ in contrast is verx high order ( Moridis \& Kansa (1993)). They have discussed the advantages/disadvantages associated with finite difference, finite element and HQ methods.

In the present paper, we have applied Mo scheme to solve the two-dimensional elliptic partial differential equations. Laplace, Poisson and Biharmonic equations have been taken as the test exanples. The gethod is also applied successfully in a problen with the curve boundary.

Appl 1 cat 1 on of MQ methind.

$$
\begin{aligned}
& \text { Consider an elliptic POE of the form. } \\
& \nabla^{2}=s(\vec{x})
\end{aligned}
$$


where $\nabla^{2}$ is the Laplacian operator, $\vec{x}$ is the space vector, $s$ is a known function of space coordinates and $f$ is an unknown function of $\vec{x}$.

The equation (1) is subject to Dirichlet and/or Neumann boundary conditions:

(a) $f=f_{1}(\vec{x})$ on $r_{1}$

(b) $\hat{n} \cdot \nabla f=f_{2}(\vec{x})$ on $\Gamma_{2}$

where $\hat{n}$ is the outward unit normal and $\Gamma_{1}$ and $\Gamma_{2}$ are the boundaries of the domain, $f_{1}$ and $f_{2}$ are known functions of $\vec{x}$. MQ method assumes that any function can be expanded as a finite series of basis functions (Kaisa (1990b) \& Hardy (1990)). The expansion used is the Madych \& Nelson's (1989) expansion with an appended constant. i.e...

$f(\vec{x})=a_{1}+\sum_{j=2}^{N} a_{j} \hat{g}\left(\vec{x}-\vec{x}_{j}\right)$

where $N$ is the total number of data points under consideration, $a_{j}^{\prime} s$ are the coefficients and the functions $\hat{g}$ are defined by

$\hat{g}\left(\vec{x}-\vec{x}_{j}\right)=g-q$

in which

$g_{j}=g\left(\vec{x}-\vec{x}_{j}\right)=\left[\left(\vec{x}-\vec{x}_{j}\right)^{2}+r_{j}^{2}\right]^{1 / 2}$

where $\left(\vec{x}-\vec{x}_{j}\right)^{2}$ is the square of the Euclidean distance $R$ in $R^{d}$ and $r_{j}^{2}>0$ is the shape function. Hagen Kansa (1993) have studied 
the role of the parameter $\left(r_{j}^{2}\right)$ in the multiquadric function. Carlson \& Foley (1991) have used a constant value of $r$ in the equation (5). Kansa $(1988,90 a \& b)$ has found numerically that the following power law function increases accuracy significantly:

$$
r_{j}^{2}=r_{m}^{2}\left(\frac{r_{n}^{2}}{r_{m}^{2}}\right)^{\frac{j-1}{N-1}} ; j=1,2 \ldots \ldots . . . N
$$

in which $r_{m}$ and $r_{n}$ are input parameters.

Spatial partial derivatives of the function in (3) are obtained by differentiating the spatial basis functions. Substitution of the function ( 3 ) and its partial derivatives in equation (1) together with the boundary conditions ( $2 a \& b)$, yields a system of linear algebraic equations which can be solved for the coefficients $a_{j}$.

We illustrate the method for a two dimensional problem. The derivatives are given by :

$\left.\frac{\partial f}{\partial x}\right|_{i=2} ^{N} x_{i} y^{-}=\sum_{j=2}\left(\frac{x_{i}-x_{j}}{g_{i j}}-\frac{x_{i}-x_{1}}{g_{i 1}}\right)$

$\frac{\partial f_{j}}{\left.\partial x_{i}+y_{i}\right),}=\sum_{j=2}^{N} a_{j}\left(\frac{y_{i}-y_{j}}{g_{i j}}-\frac{y_{i}-y_{1}}{g_{i 1}}\right)$

$\bar{\partial}_{\partial x^{2}}^{2}\left(x_{i}+y_{i}\right)=\sum_{j=2}^{N}{ }^{2} j\left(\frac{1}{g_{i j}}-\frac{\left(x_{i}-x_{j}{ }^{2}\right.}{g_{i j}^{2}}-\frac{1}{g_{i 1}}+\frac{\left(x_{i}-x_{1} 3\right.}{g_{i 1}^{2}}\right)$ 
$\left.\frac{\partial^{2} f}{\partial y^{2}}\right|_{\left(x_{i}, y_{i}\right)}=\sum_{j=2}^{N} a_{j}\left[\frac{1}{g_{i j}}-\frac{\left(y_{i}-y_{j}\right)^{2}}{g_{1 j}^{3}}-\frac{1}{g_{i 1}}+\frac{\left(y_{i}-y_{i}\right)^{2}}{g_{i 1}^{3}}\right]$

in which

$$
g_{i j}=\left[\left(x_{i}-x_{j}\right)^{2}+\left(y_{i}-y_{j}\right)^{2}+r_{j}^{2}\right] .1 \underline{1} i_{i} \underline{j} N .
$$

Substituting the expansion of $f$ (equation 3 ) and its derivatives (equation 7 ) in the two dimensional version of equation (1), we find for each of the interior node $i$,

$$
\sum_{j=1}^{N} p_{i j} a_{j}=b_{i}
$$

in which

$$
\begin{aligned}
& p_{i 1}=0 \\
& p_{i j}=2\left(g_{i j}^{-1}-g_{i 1}^{-1}\right)-g_{i j}^{-3}\left\{\left(x_{i}-x_{j}\right)^{2}+\left(y_{i}-y_{j}\right)^{2}\right\}+ \\
& g_{i 1}^{-3}\left\{\left(x_{i}-x_{1}\right)^{2}+\left(y_{i}-y_{1}\right)^{2}\right\} \quad ; 2 \leq j \leq N \quad(10) \\
& b_{i}=s\left(x_{i} \cdot y_{i}\right) \\
& \text { If a point i lies on the boundary, it may satisfy either }
\end{aligned}
$$

Dirichlet or a Neumann boundary condition.

Case I : With Dirichlet condition $f=f_{1}$, coefficients in i-th equation (9) will be 


$$
\left\{\begin{array}{l}
p_{i 1}=1 \\
p_{i j}=g_{i j}-g_{i 1} \\
b_{i}=f_{1}\left(k_{i} \cdot y_{i}\right)
\end{array} \quad 2 \leq J \leq N\right.
$$

Case II: Neumann condition $\ddot{n} \cdot \nabla f=f_{2}$.

(a) If the point i lies on the boundary on which the normal is in the direction of $\%$, coefficients in $i-t h$ equation (9) are given by:

$$
\begin{aligned}
& p_{i 1}=0 \\
& p_{i j}=\left(x_{i}-x_{j}\right) / g_{i j}-\left(x_{i}-x_{1} / / g_{i 1} \quad 2 \leq j \leq N\right. \\
& b_{i}=f_{2}\left(x_{i} / y_{i}\right)
\end{aligned}
$$

(b) If the point i lies on the boundary on which the normal is - in the direction of $y$ r coefficients in $i-t h$ equation (9) are given by :

$$
\begin{aligned}
& p_{i 1}=0 \\
& p_{i j}=\left(y_{i}-y_{j}\right) / g_{i j}-\left(y_{i}-y_{1}\right) / g_{i 1} \quad 2 \leq j \leq N \\
& b_{i}=f_{2}\left(x_{i}, y_{i}\right)
\end{aligned}
$$

For all the grid points, equation (9) forms a system of linear algebraic equations of the form:

$$
P A=B
$$

in which $P=\left(p_{i j}\right)$ is the square matrix of order $N_{2}$ 
$A=\left(a_{1}, a_{2}, \ldots a_{N}\right)^{T}$ is a column vector of unknown coefficients and $B=\left(b_{1}, b_{2} \cdots b_{N}\right)^{\top}$. The elements of $P \& B$ are defined by the relations $(10-13)$.

Kansa (1990) and Dubal (1992) have discussed the nature of the system of equtions (14). Here, we have solved it successfully using Gauss elimination method with total pivoting.

The mean square error ( $E$ ) is computed from:

$$
E=\frac{1}{N} \sum_{i=1}^{N}\left(f_{i}-\ddot{f}_{i}\right)^{2}
$$

where $f_{i}$ is the computed value of $f$ using Me wethod and $\ddot{f}_{i}$ is the known value of $f$ at the point $i$. It may be known either analytically or computationally by means of some other methods such as finite differences, finite elements etc.

He have used the double precision FORTRAN on ICL 3980 computing wachine at IIT Delhi.

\section{Examples:}

In the following, we present the various examples for determining the solution of elliptic PDEs using me method.

(A) Laplace equation in a square:

Consider a Laplace equation

$$
f_{x x}+f_{y y}=0
$$

in a square domain $R(0<x<1,0<y<1)$ with the conditions:

(i) $f=0$ along the edges $x=0 ; y=1 \& x=1$

(ii) $f=$ To along $y=0$ 
In (16), subscripts " $x \times$ " " $y y$ " denote the second partial derivatives with respect to $* \&$ y respectively. $T_{0}$ is a constant. The equation (16) with the boundary conditions (17) describes the steady-state temperature distribution in a square plate, one side of which is maintained at a constant temperature $T_{0}$ with the other three sides are maintained at $0^{\circ}$

An analytical solution of (16) with the boundary conditions (17) is given by (Carslaw \& Jeager (1976)) :

$f(x, y)=,\frac{4 \pi 0}{\Pi} \sum_{n=0}^{\infty} \frac{1}{(2 n+1)} \begin{gathered}\sin (2 n+1) \Pi^{x} \\ \operatorname{cosech}(2 n+1) \Pi(1-y)(2 n+1) \Pi\end{gathered}$

The problem described by equation (16) with the boundary conditions (17) becomes a particular case of the problem (1-2) with $s(x, y)=0$ and $f_{1}=T_{0}$ with Dirichlet condition only. We use the me method discussed in the earlier section. The computation has been done with $T_{0}=100$ for different number of grid points (N). For 81 grid points at a unifor spacing $\Delta x=\Delta y=0.125$, we found the mean square error $=0.682$ with a maximun error $|3.441|$. In the next case, we have removed the grid points lying on the lines $y=0.375$ and $y=0.625$ resulting in $N=63$. Further, the grid points are reduce to 49 in the domain by deleting the points on the vertical lines $x=0.375 \& x=0.625$. By removing the additional grid points from the line $y=0.75$, we have 42 points in the domain.

Also.- we have obtained the solution for scattered data points 30.33 \& 35 respectively, and for 25 (i.e.. $5 \times 5$ ) unifor grid 
points. Table 1 provides the information about be number of boundary points, number of interior points, the value of the input parameters R. \& Rn, mean square error \& the maximum absolute error for each of the test run. Table 1 shows that mean square errors are not significantly different in all the cases. As an illustration, the computed results for $N=42$ are given in Table 2 \& Fig.1. There is a reasonably good agreement between the computed \& exact values, considering that over the unit square the values varying 0 to 100 , except at two points where the maximum error is about $|3.68|$.

The maximum error is relatively higher and is introduced at the grid point near the point of intersection of the sides with temperatures $T_{0}=100^{\circ} \& T=0^{\circ}$. Thus, the large error is because of discontinuity in temperatures $T=100^{\circ} \& T=0^{\circ}$. It resains of the same order even with the increase in the number of grid points (table 1$)$.

(B) Poisson equation in a elliptic domain :

$$
\begin{gathered}
\text { Consider a Poisson equation } \\
f_{x x}+f_{y y}=-2 \\
\text { in an ellipse with major \& minor seniaxes } \alpha \text { and } \beta \text {. }
\end{gathered}
$$

Equation (19) is subject to the condition that $f$ vanishes on the surface of the ellipse.

$$
\frac{x^{2}}{a^{2}}+\frac{y^{2}}{\beta^{2}}=1
$$

Since the solution of (19) with (20) is expected to be symeetric with respect to $x$ and $y$, it is sufficient to solve the equation (19) in the first quadrant of the elipse and accordingly. wo consider the equation (19) with the following 
Neumann \& Dirichlet boundary conditions :

$$
f_{x}=0 \text { at } x=0
$$

(ii)

$$
f_{y}=0 \text { at } y=0
$$

(iii)

$$
f=0 \text { on the curve surface }
$$

The equation (19) provides a function $f$ frow which the angle of twist of a cylindrical shaft of an elliptical cross-section under torsion can be calculated (Hughes \& Gaylord (1964)).

An analytical solution of (19) with $(20)$ is

$$
f(x, y)=-\left[\frac{x^{2}}{\alpha^{2}}+\frac{y^{2}}{\beta^{2}}-1\right)\left[\frac{\alpha^{2} \beta^{2}}{\alpha^{2}+\beta^{2}}\right]
$$

The solution of the problen (19) with (21) can be found as a particular case of the problem $(1-2)$ with $s(x, y)=-2, f_{1}=0$ and $f_{2}=0$.

For $\alpha=10, \beta=8$ and $N=28$ (figure 2 ), the results are presented in table 3 . The function varies from 39 to 0 in the domain with $E=2.92 \times 10^{-8}$. The maximum error is found to be $\left|3.574 \times 10^{-4}\right|$ which is $0.0025 \%$ of the exact functional value. The computed values are close to the exact values of the function. Me method provides the accuracy upto five significant decimal places. Computations have also been done with different sets of data points and the results are found to be very good. For $N=87$, the maximum error is found to be $16.68 \times 10^{-5}$ which is $0.00027 \%$.

For $\alpha=2, \beta=1$, the function varies from 0.8 to 0 . Again, we obtain an accurate solution with $N=28$ not only at the interior points as well as on the boundary points with $E=2.96 \times 10^{-10}$ and aximun error $=14.8 \times 10^{-5}+$. 
The no aethod provides an excellent approximate solution. The curve boundary is accounted in this method. in a natural way through the location of the boundary posnts whereas in finite difference method, an interpolation technique is required for expressing boundary points in terms of grid points.

(C) Poisson Equation in a rectangle with a constant $r$ ight hand side :

$$
\begin{aligned}
& \text { Consider a Poisson equation } \\
& \qquad f_{x x}+f_{y y}=Q
\end{aligned}
$$

where $\theta$ is a constant.

The equation (23) describes the steady state transport of oxygen in a slab of tissue with a constant oxygen consumption rate (Sharan et al (1991)). This also governs the steady state temperature distribution in a rectangular region with the heat loss (production) at a constant rate $Q(-Q)$ (Carslaw \& Jeager $(1976))$. If $\theta=\frac{q}{D}$ in which $D$ is the flexural rigidity of a thin plate subject to a normal load $q$ per unit area, the equation (23) arises by splitting a biharmonic equation (see example(E)) into two successive Poisson equations.

The boundary conditions for equation (23) are

$$
f=0 \text { along the four edges }(x \pm a, y= \pm b)
$$

of the rectangle with sides a \& b.

The solution of (23) with boundary conditions (24) will be symmetric with respect to $x \& y$ and thus, we solve the equation (23) in the region $(0<x<a, 0<y<b)$ with the boundary conditions :

(i) $\quad f_{x}=0$ at $x=0$ 


$$
t=0 \text { at } x=a
$$

(iii)

$$
f_{y}=0 \quad \text { at } y=0
$$

(iv)

$f=0$ at $y=a$

An analytical solution of the problem $(23-24)$ is given by (Carslaw \& Jeager(1976)) :

$$
\begin{array}{r}
f(x, y)=\frac{160 a^{2}}{n^{3}} \sum_{n=0}^{\infty} \frac{(-1)^{n} \cos \left[(2 n+1) \cdot \pi_{x / 2 a}\right] \cosh [(2 n+1) \pi y / 2 a]}{(2 n+1)^{3} \cosh [(2 n+1) \pi b / 2 a]} \\
-\frac{\theta}{2}\left(a^{2}-x^{2}\right) \quad \text { (26) }
\end{array}
$$

The solution of (23) with (25) can be found similar to the problew $(1-2)$ with $s(x, y)=Q, f_{1}=0$ and $f_{2}=0$. The domain has been normalized to a unit square with a \& b. For the computation. we have taken $q=20,0=2.1978 \times 10^{4}, a=20$ and $b=20$. We have performed a series of calculations with 25 (i.e... 5x5) grid points with a unifor spacing $\Delta x=\Delta y=0.25$ \& with 35 scattered data points. Values of the input parameters, mean square error, and maximum absolute error are summarized in Table 4. Table 5 shows that conputed solution compares well with the exact solution for 35 scattered data points with $E=2.03 \times 10^{-7}$. The function varies from $=0.11$ to 0 . The maximum error is found to be 1.35 $\times 10^{-3}$ which is less than $2.3 \%$. He have also obtained a good solution with $N=35$ for $q=1$.

(D) Poisson Equation in a rectangle with right hand side as a function of space coordinates :

Consider a Poisson equation in a rectangular plate $(-a<x<a,-b<y<b):$

$$
f_{x x}+f_{y y}=g(x, y)
$$


where 8 lo a known function of $x$ \& $y$. Iaportant applicatlone occur: (a) in flusd dynamics with $f=$ stream function and $g=$ -vorticity, (b) in electrostatics, with $f=$ electric potential and -g represents the rat 10 of charge density to dielectric constant. (c) in heat transfer, with $f=$ temperature and $g$ is the loss/generation of heat, (d) in wass transfer with $f=$ concentration and $g$ is the source/sink term.

Here we consider $g$ of the form of (26). The equation (27) arises as the second Poisson equation in the splitting of biharmonic equation (see, example (E)) describing the transverse deflection of a thin plate.

Again, with the boundary conditions (24), the solution of (27) is expected to be symmetric in $x$ and $y$ and thus, we consider the region $(0<x<a, 0<y<b)$ with the boundary conditions (25).

An analytical solution of (27) with boundary conditions (24) is obtained in form:

$$
\begin{gathered}
-f(x, y)=\frac{a}{a} \sum_{n=0}^{\infty} \frac{(-1)^{n}}{a_{n}^{5}}\left[2+a_{n} y \frac{\sinh a_{n}^{y}}{\cosh \alpha_{n}^{b}}-\left[2+\frac{a_{n}^{b} \sinh \alpha_{n}^{b}}{\cosh a_{n}^{b}}\right]\right. \\
\left.\frac{\cosh \alpha_{n} y}{\cosh \alpha_{n}^{b}}\right] \cos a_{n} x
\end{gathered}
$$

in which

$$
a_{n}=\frac{(2 n+1) \Pi}{2 a}
$$

We normalize the domain to a unit square with a b. The values 
of G, D, a, b are the ane ae in the previous example. Table 6 contains the values of the input parameters, mean square error and maximum absolule error for $q=20$ and $q=1$ with different $N$.

The results presented in table $7 a$ for $q=20$ with $N=35$ are very good. considering the points are scattered. In most of the runs (table 6), the relative percentage error

$$
\left(100 x \max \left|f j-\ddot{f}_{j}\right| / \grave{f j}_{j}\right)
$$

is less than one percent.

The table $7 b$ shows that the computed values are close to the analytical values for $q=1$ with $N=30$. The maximum error is 6.6 $\times 10^{-4}$ which is approximately $0.02 x$ of its analytical value.

Thus, the ne method provides an accurate solution to the equation (27) with the boundary conditions (24).

\section{(E) Biharmonic Equation:}

The transverse deflection $(f)$ of a thin rectangular plate with sides $2 a$ and $2 b$, of flexural rigidity 0 subject to normal load q per unit area is governed by

$$
\nabla^{4} f=f_{x x x x}+2 f_{x x y y}+f_{y y y y}=\frac{q}{D},(-\bar{a}<x<a,-b<y<\bar{b})
$$

The equation (29) is subject to the boundary conditions $f=0$ and $f_{n \eta}=0$ along its four edges, where $n$ denotes the normal to the boundary.

By introducing the variable $u=\nabla^{2} f$, the problem (29) amounts to solving Poisson's equation twice in succession:

$$
\begin{aligned}
& \text { (i) } u_{x x}+u_{y y}=\frac{q}{D} ;-a<x<a,-b<y<b \\
& \text { with } u=0 \text { along the four edges. }
\end{aligned}
$$




$$
\begin{aligned}
& \text { and } \text { (ii) } \underset{K K}{ }+f_{y y}=u(x, y) ;-a<\dot{x}<a,-b<y<b \\
& \text { with } f=0 \text { along the four edges } x= \pm a \quad \& y= \pm b .
\end{aligned}
$$

Notice that the problems (30) and (31) are the same to the problems (23) \& (27). In order to obtain f from (31), the solution of (30) w1 be used as the input. The relation (28) is the analytical solution of the biharmonic equation (29). The values of $q$, $D$, a \& $b$ are the same as given in examples (C)\& (D). As in the previous cases, the domain was normalized to a unit square with reference to $a$ and $b$. The values of the inputs parameters, mean square error and the maximum absolute error for different $N$ and $q$ are given in table 8 .

The solution of the biharmonic equation (29) for $q=20$ is presented in table 9 for 35 scattered data points and in figure 3 for 25 uniform points. There is a good agreement between the computed and analytical values(table 9 fig 3 ).

The computed values are found to be closed (Table 10 ) to the exact values for $q=1$ with 25 uniform grid points. Notice that the accuracy of the solution of biharmonic equation computed in two stages using equations (30\&31) and in single stage from equation (27) is found to be almost same.

Conclusions :

We have applied successfully he method to compute the solution of elliptic partial differential equations with Dirichlet and/or Neumann boundary conditions. The data points in arbitrary locations with an arbitrary ordering allows us to obtain an accurate solution. The method is shown to work well for Laplace. 
Polason and Biharmonic equations. When comparing the MQ computed values with the exact solution, the agreement is found to ve very good. For the summation of the series in (18), (26) and (28), sufficiently large number of terms (n) are considered such that the absolute value of nth term is less than or equal to $10^{-10}$.

In the first example, the error was relatively higner on the grid points near the point of intersection of the sides with different temperatures.

Me method performs well for the problews with curve boundary. The curve boundary is accounted, here, in a natural way through the location of the data points unlike finite difference method in which an interpolation technique is required to express a boundary point in terms of grid points.

We have shown $A Q$, is an excellent approximation scheme for solving a biharmonic equation. The solution of this equation obtained in two stages is approximately the same as obtained in single stage.

Because the coefficient atrix-is full, *ansa (19906) has suggested domain deconposition and blending (Foley (1992)). However, this will increase the computational conplexities. Dubal et al (1992) have suggested the use of singular value decomposition (Press et al(1986)) for solving the resulting system of algebraic equations. However, we have observed that in the examples considered here the Gaussian elimination with total pivoting gives the better results than the singular value decomposition.

The accuracy of the computed solution depends on the choice of 
the parameters $R_{m} \& R_{n}$. In the present study, their values are chosen by experimentation. Madych (1992) has shown that mult zquadrics converge very quickly and Baxter (1992) has found that the He schene converges rapidly for a constant value of $R^{2}$ if $R^{2}$ goes to infinity. Such large values forces severe ill-conditioning. Carlson \& Foley (1991) have estimated a good value for the constant $R^{2}$ in the Me equation. Kansa (1990a\& b) has shown that the power law seems to work very well. Hager \& Kansa (1993) use brent algorithm to find the optimum constant $R$ or the shape parameters $R_{m} \& R_{n}$. We have also made an attempt to obtain these shape parameters using nonlinear optimization technique suggested by Marquardt (1963). However, the determination of these parameters requires the prior knowledge of the exact solution. Recently, Galperin and Zheng (1993) and Galperin (1990) have suggested a method based on global optimization techniques which way be used for the estimation of shape parameters in case the exact solution is not known.

Quite often, the solution is very sensitive to $R m \& R n$ and a slight variation in $R_{m} \& R_{n}$ may change significantly the mean square errors. Further, there is no systematic way to determine the choice of $R_{m} \& R_{n}$ like the finite difference methods where for consistent a stable approximation scheme, a decrease in the grid spacing yields to a more accurate results. Thus, it is necessary to develop a suitable procedure for estimating Rm \& Rn in terms of function values. $N$ and location of grid points.

Recently. Galperin and Zheng (1993) have developed a new method for the solution and control of PDEs via global optimization methods (Galperin(1990) and Galperin and Zheng $(1990)$ ). By formulating any PDE problew as an optiaization one 
(Galperin and 2hene (1993)), even if the exact solution io not known, one can readily optimize the chosces of the me shape parameters, and also can deal more effectively wath the nonlinear POEs. Such an approach would elliminate the guess work in dealing with the shape parameters and the delta algorithm calperin $(1990)$ ) is expected to provide fast convergence with a minimua number of evaluations. Thus, one of the future direction of the research is to apply global optimization techniques for the solution of PDEs with MQ.

In the subsequent studies, we propose to apply mo scheme to more complicated physical problems involving arbitrary geometrical configuration. interface conditions and nonlinearities. 


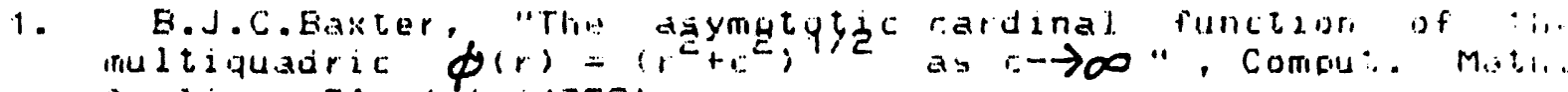
Affilic., E4, 1-6 (1992).

E. R.E.Carlson and T.A.Foley, "The paraneter $R E$ multiquadri- interpolation". Computera Math. Applic..21. 2942 (1991).

3. H.S.Carglaw and J.C.Jaeger, "Heat Conduction in Solidg", Oxford Univeralty Preas, Oxtord 1976.

4. M.R.Dubal, "Conatruction of theet-dimensional black-hole initial data via multiquadrics", Phys. Rev. D.,45.1178-1187 $(1992)$.

5. M.R.Dubal, S.R.01iveira and R.A.Matzner," Solution ot elliptic equations in numerical relativity usine multiquadrica". In: Approaches to Numerical Relativity edited by R.D. Inverno. Cambridee University Press. Cambridee, Eneland (1992).

6. T.A.Foley, "The map and blend acattered data interpolant on a spherg". Comput. Math. Applic..24, 49-60 (1992).

7. R.Franke, "Scattered data interpolation: teats of some methods" Math.Comp..38,181-200 (1982).

8. E.A.Galperin. "The Cubic Aleorithn for Optimization and Control". NP Research Publ Montreal, 1990.

9. E.A.Galperin and Q.Zhone. "Solution and control of PDE via

- Global optimization methods." Comput.Math. Applic..25, 103$118(1993)$.

10. E.A.Galperin and Q.Zhene. "Now Theory of Continuous Games" NP Regearch Publ Montreal, 1990.

11. R.E.Hacen and E.J.Kansa, "Studiog of tho R parametor in the multiquadric function appliod to eround water pumpine". Accopted for publication in the spocial isaue for Advances in the Theory Applications of Radial Bagia Functions. Int J Computer Simulation Modelline (1993).

12. R.L.Hardy,"Thoory and applications of the multiquadricbiharmonic mothod. 20 Years of discovery 1968-1988". Comput.Math. Appl.. 19, 163-208 (1990).

13. U.F.Huches \& E Gaylord, "Basic Equationa of Eneinoering Scionce". Schaum Publishine Company. Now York 1964.

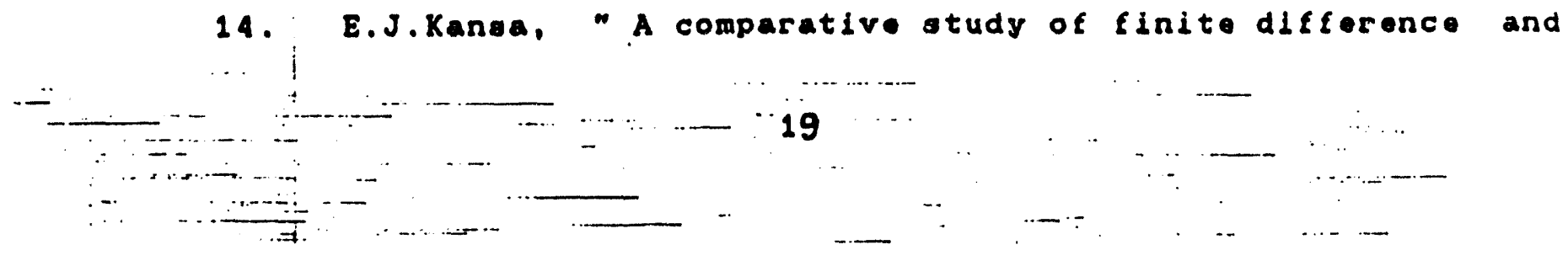


multiquadric schemes for the Euler equations". Simulation. 51.180-183 (1988).

15. E.J.Kanga." Multiquadrica - A scattered dutid approximation scheme with applications to Computational Fluid Dynamics I: surface approximations and partial derivative ostimates". Comput. Math. Applic.. 19. 127-154 (1990).

16. E.J.Kansa," Multiquadrica - A scattered data approximation scheme with applications to Computational Fluid Dynamiss I I : solutions to parabolic, hyperbolic, and elliptic partial differential equations", Comput. Math. Applic.. 19.146-161 $(1990)$.

17. J.Kevorkian and J.D.Cole, "Perturbation methods in Applied Mathematics". Sprineer. New York 1981.

18. W.R.Madych, "Miscellaneous error bounds for multiquadric and related interpolators", Comput. Math. Applic..24, 121$138(1992)$.

19. W.R.Madych and S.A.Neloon, Multivariate interpolation and conditionally positive definite functions". Approx. Theo. App1..4. 77-89 (1989).

20. W.R.Madych and S.A.Nelson, "Multivariate interpolation and conditionally positive definite functions II". Math. Comput., 54 211-230 (1990).

21. A.Makroglou, "Radial bagis functiong in the numerical solution of Fredholm integral and integro-differential equations". In: Advances in Computer Methods for Partial Differential Equations-VII, pp 478-484, Editors R. Vichnevetsky, D. Knight \& G. Richter. Proc of the 7 th IMACS conference on Computer Methods for PDEs- New Brunswich. NJ, USA, June 22-24 (1992).

22. D.M.Marquardt, An aleorithm for least aquare estimation of nonlinear parameters. J Soc Ind Appl Math, 11.431-441 (1963).

23. C.A.Micchelli. "Interpolation of scattered data: distance matrices and conditionally positive definite functions", Const. Approx., $2,11-22$ (1986).

24. G.Moridia \&.J.Kanaa, "The Laplace tranaform multiquadric method: a hiehly accurate scheme for numerical solution of partial differential equations". Comput. Math. Applic. (submitted).

25. A.H.Nayteh. "Perturbation Methods". Wiley Intergcience, New York 1973.

26. J.Y.Parlane, "Theory of water movements in soils I: One dimengional absorption". Soil Sci..111.134-170 (1971). 


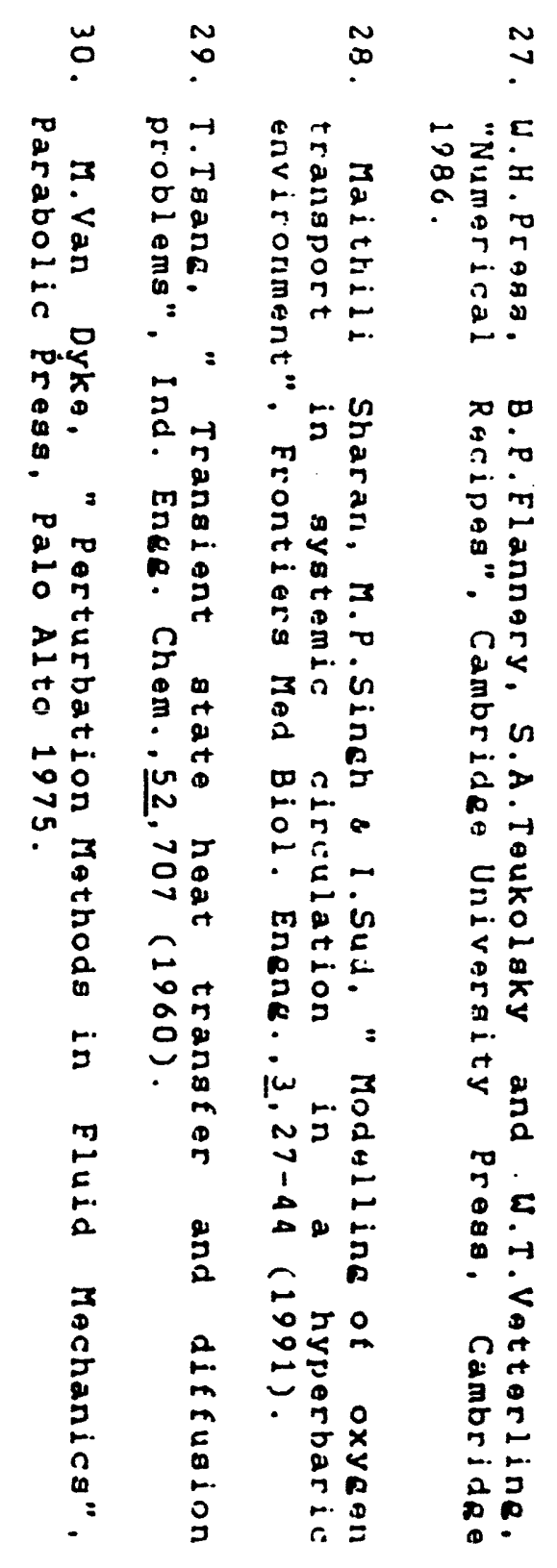


Table 1. Values of input parameters, mean equare error and maximua aboolute error for Laplace equation.

S. No. Total number Number of Number of Rm Rri Meari Maxl mum of polnts boundary interior Points poirits square Absilute errour error

\begin{tabular}{lllllllll}
1. & 61 & 32 & 40 & .22 & 11 & .682 & 3.44 \\
2. & 63 & 28 & 35 & .12 & 121.57 & 4.02 \\
3. & 49 & 24 & 25 & .14 & 12 & .832 & 3.65 \\
4. & 42 & 22 & 20 & .14 & 10 & 1.08 & 3.68 \\
5. & 25 & 16 & 0 & 0.4 & 50 & 1.68 & 3.88 \\
6. & 30 & 18 & 12 & 1.3 & 102.68 & 4.71 \\
7. & 33 & 18 & 14 & 0.068503 .53 & 5.23 \\
8. & 35 & 15 & 20 & .7 & 103.35 & 0.0 \\
\hline
\end{tabular}



TABLE 2: COMPUTED AND E\%AI.: "OLIITIIIN OF THE LAPLAIE
EQUATION IN A SQUA.

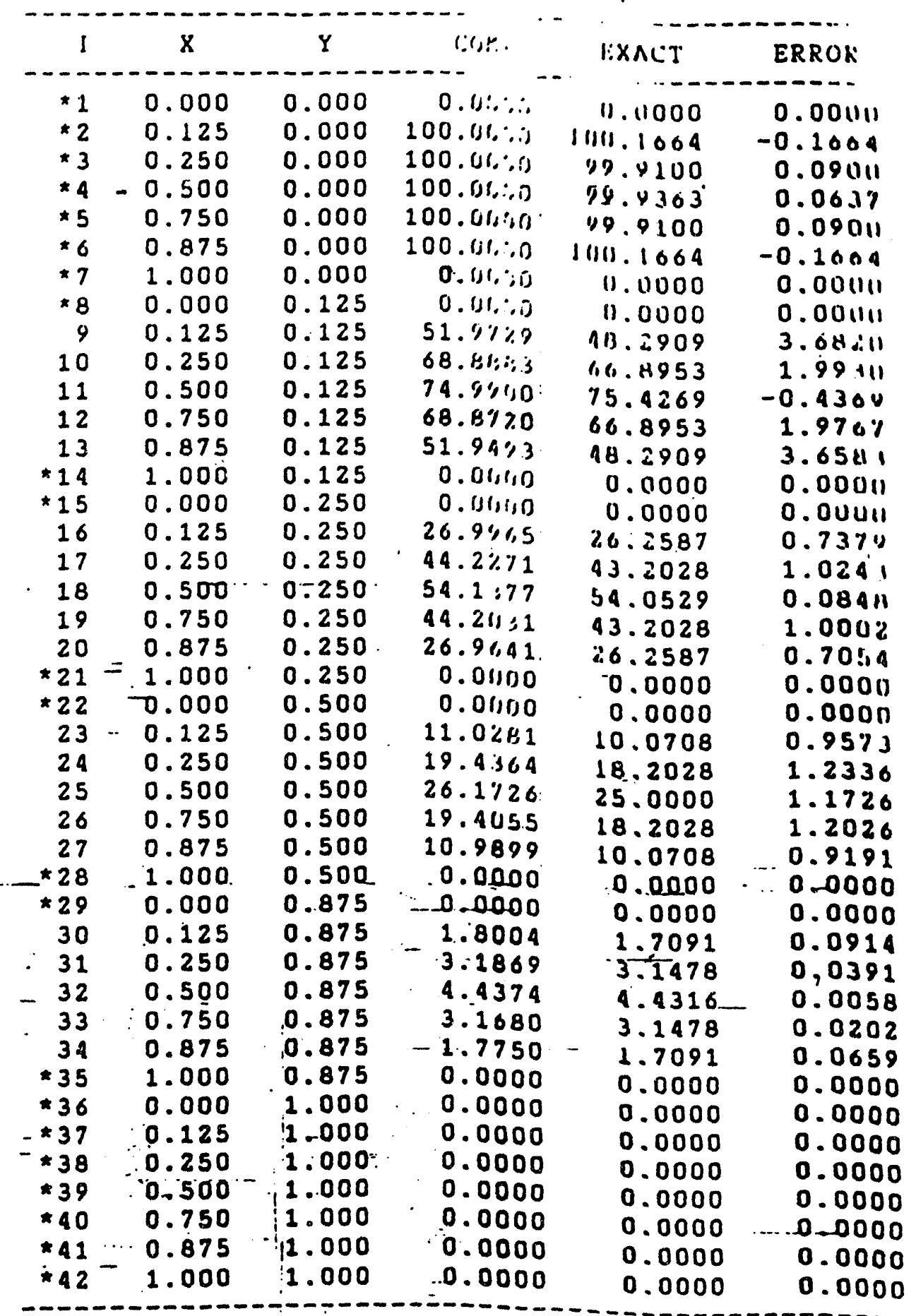

* Boundary pointa. 
TABLE 3: COMPUTED AND EXACT SOLUTION OF THE POISSON EQUATION IN AN ELLIPSE. $\alpha=10, \beta=8, N=28, R m=55, R n=4$.

\begin{tabular}{|c|c|c|c|c|c|}
\hline I & $x$ & $Y$ & COMP & EXACT & ERROR \\
\hline$* 1$ & 0.000 & 0.000 & 39.0247 & 39.0244 & 0.000 .3 \\
\hline *2 & 0.000 & 1.600 & 4635 & 37.4634 & 0.0001 \\
\hline$* 3$ & 0.000 & 00 & 29.2681 & 29.2683 & -0.0001 \\
\hline$\star 4$ & 0.000 & 6.400 & 14.0484 & 14.0488 & -0.0004 \\
\hline$* 5$ & 0.000 & 8.000 & 0.0000 & 0.0000 & 0.0000 \\
\hline$\star 6$ & 2.000 & 0. & 37. & 37. & 03 \\
\hline$\star 7$ & 4.000 & 0.000 & 32.7808 & 32.7805 & 0.0003 \\
\hline$\star 8$ & 6.000 & 0.000 & 24.9759 & 24.9756 & 0.0003 \\
\hline$* 9$ & 8.000 & 0.000 & 14.0490 & 14.0488 & 0.0002 \\
\hline 10 & 10.000 & 0.000 & 0.0000 & 0.0000 & 0.0000 \\
\hline 11 & 2.000 & 1.600 & 35.9026 & 35. & 1001 \\
\hline 12 & 4.000 & 1.600 & 31.2197 & 31.2 & 0.0002 \\
\hline 13 & 6.000 & 1.600 & 23.4148 & 23.4 & 0.0002 \\
\hline 14 & 8.000 & 1.600 & 12.4879 & 12.4 & 0.0001 \\
\hline 15 & 2.000 & $4: 0$ & 27.7072 & 27.7 & -0.0001 \\
\hline 16 & 4.000 & 4.000 & 23.0244 & 23.0244 & 0.0000 \\
\hline 17 & 6.000 & 4.000 & 15.2195 & 15.21 & 0.0000 \\
\hline 18 & 8.000 & 4.000 & 4.22927 & 4.2 & 1000 \\
\hline 19 & 2.000 & 5.600 & 18.3412 & 18.3415 & -0.0002 \\
\hline 20 & 4.000 & 5.600 & 13.6584 & 13.6585 & -0.0001 \\
\hline 21 & 6.000 & 5.600 & 5.8536 & 5.8537 & 0.0000 \\
\hline 22 & 9.798 & 1.600 & 0.0000 & 0.0000 & 0.0000 \\
\hline 23 & 8.660 & 4.000 & 0.0000 & 0.0 & 0.0000 \\
\hline 24 & 7.141 & 5.600 & 0.0000 & 0.0000 & 0.0000 \\
\hline 25 & 2.000 & 7.838 & 0.0000 & 0.0 & 0.0000 \\
\hline$: 6$ & 4.000 & 7.332 & 0.0000 & 0.0 & 0.0000 \\
\hline & 6.000 & 6.400 & 0.0000 & 0.0000 & 0.0000 \\
\hline $2 \varepsilon$ & 8.000 & 4.800 & 0.0000 & 0.0000 & 0.0000 \\
\hline
\end{tabular}

* Boundary pointa. 
Table 4 : Val cues of the 1 roput parameters, Moan Square ardor (ES, alosiol ute maxi mum error $t$ is different $N$ for $\nabla^{2} t=0$.

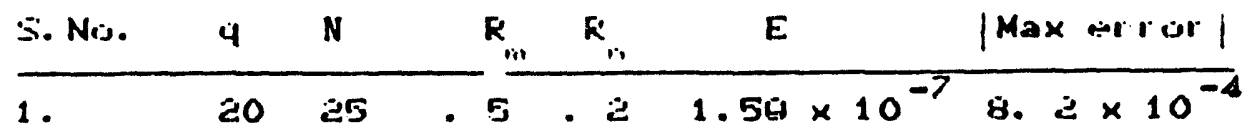

2. $2035.0 .12 .03 \times 10^{-7} 1.35 \times 10^{-3}$

3. $125 \cdot 5 \cdot 23.98 \times 10^{-10} 4.1 \times 10^{-5}$

4.

$135.7 .11 .28 \times 10^{-9} 1.46 \times 10^{-4}$

25 
TABLE 5: COMPUTED AND EXACT SOLUTION OF THE POISSON EQUATION $f \times X+f Y Y=q / D$ IN WHICH $a$ IS A CONSTANT. $q=20, N=35, R m=0.9, R_{n}=0.1$.

\begin{tabular}{|c|c|c|c|c|c|}
\hline I & $x$ & $\mathbf{Y}$ & COMP & EXACT & ERROR \\
\hline *1 & 0.000 & 0.000 & -0.10644 & -0.10727 & $0.000 ?$ \\
\hline$\star 2$ & 0.000 & 220 & -0.10222 & -0.10282 & 0.00000 \\
\hline$* 3$ & 0.000 & 0.500 & -0.08387 & -0.08348 & -0.00039 \\
\hline$\star 4$ & 0.000 & 0.700 & -0.06006 & -0.05871 & -0.00135 \\
\hline$\star 5$ & 0.000 & 1.000 & 0.00000 & 0.00000 & 0.00000 \\
\hline$\star 6$ & 0.250 & 0.000 & -0.10045 & -0.10151 & 0.00107 \\
\hline$* 7$ & 0.500 & 0.000 & -0.08334 & -0.08348 & 0.00014 \\
\hline$* 8$ & 0.810 & 0.000 & -0.04167 & -0.04048 & -0.00119 \\
\hline *9 & 0.180 & 1.000 & 0.00000 & 0.00000 & 0.00000 \\
\hline * 10 & 0.500 & 1.000 & 0.00000 & 0.00000 & 0.00000 \\
\hline ^ 11 & 1.000 & 0.000 & 0.00000 & 0.00000 & 0.00000 \\
\hline * 12 & 1.000 & 1.000 & 0.00000 & 0.00000 & 0.00000 \\
\hline * 13 & 1.000 & 0.500 & 0.00000 & 0.00000 & 0.00000 \\
\hline$\star 14$ & 1.000 & 0.700 & 0.00000 & 0.00000 & 0.00000 \\
\hline *15 & 0.800 & 1.000 & 0.00000 & 0.00000 & 0.00000 \\
\hline 16 & 0.130 & 0.260 & -0.09909 & -0.09961 & 0.00052 \\
\hline 17 & 0.310 & .420 & -0.08330 & -0.08349 & 0.00018 \\
\hline 18 & 0.770 & 0.580 & -0.03505 & -0.03496 & -0.00009 \\
\hline 19 & 0.220 & 0.730 & -0.05261 & -0.05216 & -0.00046 \\
\hline 20 & 0.930 & .180 & -0.01642 & -0.01599 & -0.00044 \\
\hline 21 & 0.860 & 0.780 & -0.01545 & -0.01538 & -0.00007 \\
\hline 22 & 0.420 & 0.910 & -0.01801 & -0.01816 & 0.00015 \\
\hline 23 & 0.510 & 0.570 & -0.05946 & -0.05961 & 0.00015 \\
\hline 24 & 0.680 & 0.820 & -0.02430 & -0.02435 & 0.00005 \\
\hline 25 & 0.720 & 0.620 & -0.03827 & -0.03824 & -0.00003 \\
\hline 26 & 0.640 & 0.330 & -0.06183 & -0.06152 & -0.00030 \\
\hline 27 & 0.840 & 0.370 & -0.03185 & -0.03147 & -0.00039 \\
\hline 28 & 0.970 & 0.680 & -0.00481 & -0.00477 & -0.00004 \\
\hline 29 & 0.270 & 0.930 & -0.01555 & -0.01554 & -0.00001 \\
\hline 30 & 0.530 & 0.110 & -0.07978 & -0.07963 & -0.00015 \\
\hline 31 & 0.150 & 0.210 & -0.10062 & -0.10126 & 0.00064 \\
\hline 32 & 0.690 & 0.950 & -0.00766 & -0.00763 & -0.00003 \\
\hline 33 & 0.480 & 0.440 & -0.07151 & -0.07163 & 0.00012 \\
\hline 34 & 0.210 & 0.570 & -0.07358 & -0.07332 & -0.00026 \\
\hline & 0.590 & .940 & -0.01061 & -0.01058 & -0.00003 \\
\hline
\end{tabular}

* Boundary pointa. 
Tabl 6 : Val ues of the 1 nput parameters, Mean square er.or. (E),

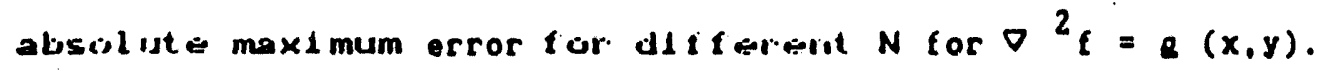

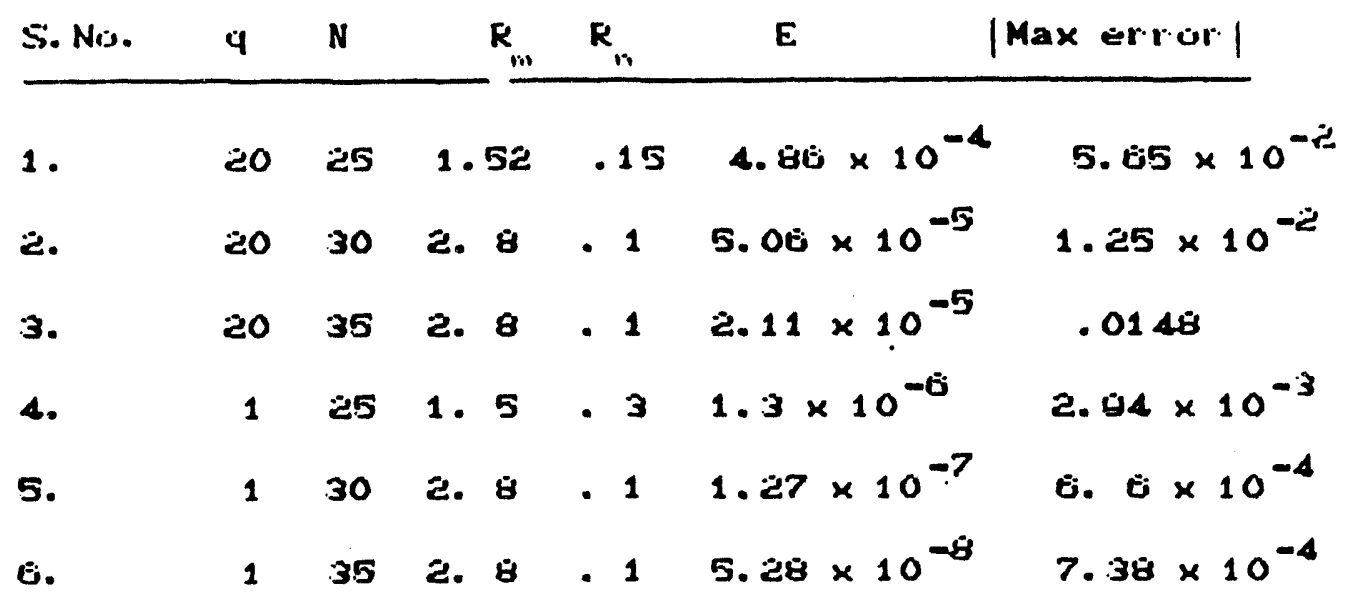


TABLE 72: COMPUTED AND EXACT SOLUTION OF THE POISSON EQUATION $E X X+\ell Y y=G(x, y)$. $q=20, N=35, R m=2.8, R n=0.1$.

\begin{tabular}{|c|c|c|c|c|c|}
\hline 1 & $x$ & $\mathbf{Y}$ & COMP & EXACT & ERROR \\
\hline & 0.000 & 0.000 & 9.46105 & 9.46366 & -0.00261 \\
\hline 2 & 0.000 & 0.220 & 8.94240 & B. 94669 & -0.00428 \\
\hline 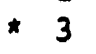 & 0.000 & 0.500 & 6.83381 & 6.84478 & -0.01097 \\
\hline 4 & 0.000 & 0.700 & 4.45365 & 4.46840 & -0.01476 \\
\hline 5 & 0.000 & 1.000 & 0.00000 & 0.00000 & 0.00000 \\
\hline 6 & 0.250 & 0.000 & 8.79259 & 8.79692 & -0.00434 \\
\hline 7 & 0.500 & 0.000 & 6.83966 & 6.84478 & -0.00512 \\
\hline 8 & 0.810 & 0.000 & 2.91293 & 2.91812 & -0.00519 \\
\hline 9 & 0.180 & 1.000 & 0.00000 & 0.00000 & 0.00000 \\
\hline$\star 10$ & 0.500 & 1.000 & 0.00000 & 0.00000 & 0.00000 \\
\hline *11 & 1.000 & 0.000 & 0.00000 & 0.00000 & 0.00000 \\
\hline * 12 & 1.000 & 1.000 & 0.00000 & 0.00000 & 0.00000 \\
\hline * 13 & 1.000 & 0.500 & 0.00000 & 0.00000 & 0.00000 \\
\hline$\star 14$ & 1.000 & 0.700 & 0.00000 & 0.00000 & 0.00000 \\
\hline * 15 & 0.800 & 1.000 & 0.00000 & 0.00000 & 0.00000 \\
\hline $\begin{array}{l}16 \\
17\end{array}$ & $\begin{array}{l}0.130 \\
0.310\end{array}$ & $\begin{array}{l}0.260 \\
0.420\end{array}$ & $\begin{array}{l}8.57076 \\
6.78130\end{array}$ & $\begin{array}{l}8.57616 \\
6.78721\end{array}$ & $\begin{array}{l}-0.00540 \\
-0.00591\end{array}$ \\
\hline $\begin{array}{l}17 \\
18\end{array}$ & 0.770 & 0.580 & 2.22804 & 2.22966 & -0.00162 \\
\hline 19 & 0.220 & 0.730 & 3.83535 & 3.84322 & -0.00787 \\
\hline 20 & 0.930 & 0.180 & 1.05650 & 1.05684 & -0.00035 \\
\hline 21 & 0.860 & 0.780 & 0.78539 & 0.78637 & -0.00098 \\
\hline 22 & 0.420 & 0.910 & 1.13562 & 1.13662 & -0.00101 \\
\hline 23 & 0.510 & 0.570 & 4.35235 & 4.35595 & -0.00360 \\
\hline 24 & 0.680 & 0.820 & 1.40578 & 1.40709 & -0.00131 \\
\hline 25 & 0.720 & 0.620 & 2.46047 & 2.46234 & -0.00187 \\
\hline 26 & 0.640 & 0.330 & 4.61329 & 4.61689 & -0.00361 \\
\hline 27 & 0.840 & 0.370 & 2.10201 & 2.10327 & -0.00126 \\
\hline 28 & 0.970 & 0.680 & 0.23959 & 0.23987 & -0.00028 \\
\hline 29 & 0.270 & 0.930 & 1.00667 & 1.00844 & -0.00177 \\
\hline 30 & 0.530 & 0.110 & 6.43731 & 6.44232 & -0.00501 \\
\hline 31 & 0.150 & 0.210 & 8.75920 & 8.76403 & -0.00482 \\
\hline 32 & 0.690 & 0.950 & 0.38783 & 0.38836 & -0.00052 \\
\hline 33 & 0.480 & 0.440 & 5.53565 & 5.53995 & -0.00429 \\
\hline 34 & 0.210 & 0.570 & 5.78024 & 5.78855 & -0.00831 \\
\hline 35 & 0.590 & 0.940 & 0.58868 & 0.58942 & -0.00074 \\
\hline
\end{tabular}

*Boundary pointa. 
TABLE 7b: COMPUTED AND EXACT SOLUTION OE THE POISSON EQUATION $E X X+f Y Y=e(x, y)$. $q=1, N=30, R_{m}=2.8, R_{n}=0.1$.

\begin{tabular}{|c|c|c|c|c|c|}
\hline I & $x$ & $\mathbf{Y}$ & COMP & EXACT & ERROR \\
\hline 1 & 0.000 & 0.000 & 0.47259 & 0.47318 & -0.00059 \\
\hline 2 & 0.000 & 0.250 & 0.43932 & 0.43985 & -0.00052 \\
\hline * 3 & 0.000 & 0.500 & 0.34184 & 0.34224 & -0.00040 \\
\hline * 4 & 0.000 & 0.750 & 0.18864 & 0.18907 & -0.00043 \\
\hline$\star 5$ & 0.000 & 1.000 & 0.00000 & 0.00000 & 0.00000 \\
\hline 6 & 1.000 & 0.000 & 0.00000 & 0.00000 & 0.00000 \\
\hline$\star 7$ & 1.000 & 0.250 & 0.00000 & 0.00000 & 0.00000 \\
\hline$\star 8$ & 1.000 & 0.500 & 0.00000 & 0.00000 & 0.00000 \\
\hline$* 9$ & 1.000 & 0.750 & 0.00000 & 0.00000 & 0.00000 \\
\hline$\star 10$ & 1.000 & 1.000 & 0.00000 & 0.00000 & 0.00000 \\
\hline$\star 11$ & 0.200 & 0.000 & 0.45114 & 0.45177 & -0.00063 \\
\hline *12 & 0.200 & 1.000 & 0.00000 & 0.00000 & 0.00000 \\
\hline$\star 13$ & 0.400 & 0.000 & 0.38796 & 0.38862 & -0.00065 \\
\hline$\star 2.4$ & 0.400 & 1.000 & 0.00000 & 0.00000 & 0.00000 \\
\hline *15 & 0.600 & 0.000 & 0.28628 & 0.28695 & -0.00066 \\
\hline$\star 16$ & 0.600 & 1.000 & 0.00000 & 0.00000 & 0.00000 \\
\hline$\star 17$ & 0.800 & 0.000 & 0.15261 & 0.15323 & -0.00062 \\
\hline * 18 & 0.800 & 1.000 & 0.00000 & 0.00000 & \\
\hline $\begin{array}{l}19 \\
20\end{array}$ & $\begin{array}{l}0.050 \\
0.130\end{array}$ & $\begin{array}{l}0.050 \\
0.200\end{array}$ & $\begin{array}{l}0.46991 \\
0.42831\end{array}$ & $\begin{array}{l}0.47051 \\
0.42881\end{array}$ & $\begin{array}{l}-0.00059 \\
-0.00050\end{array}$ \\
\hline $\begin{array}{l}20 \\
21\end{array}$ & 0.460 & 0.160 & 0.35106 & 0.35154 & -0.00048 \\
\hline 22 & 0.310 & 0.420 & 0.33905 & 0.33936 & -0.00031 \\
\hline 23 & 0.070 & 0.580 & 0.29663 & 0.29698 & -0.00035 \\
\hline 24 & 0.120 & 0.730 & 0.19946 & 0.19977 & -0.00031 \\
\hline 25 & 0.420 & 0.910 & 0.05679 & 0.05683 & -0.00004 \\
\hline 26 & 0.510 & 0.570 & 0.21766 & 0.21780 & -0.00014 \\
\hline 27 & 0.680 & 0.820 & 0.07033 & 0.07035 & -0.00003 \\
\hline 28 & 0.840 & 0.370 & 0.10504 & 0.10516 & -0.00012 \\
\hline 29 & 0.970 & 0.680 & 0.01199 & 0.01199 & 0.00000 \\
\hline 30 & 0.170 & 0.930 & 0.05292 & 0.05305 & -0.00013 \\
\hline
\end{tabular}

*Boundary pointa. 
Tables 8: Vallios ar I liee 1 riput parameters, Mearisquareserror (E',

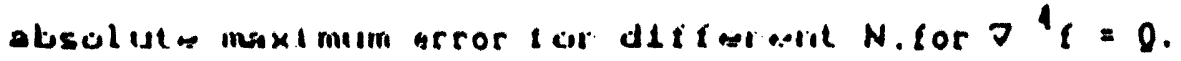
S. Ne.
R R
E.
| Max or ror |
1.

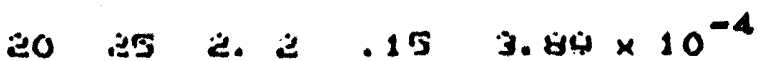
5. ت.. $\times 10^{-\ddot{2}}$
2.
20 अ 3 . 8 . $11.58 \times 10^{-4}$
3. $40 \times 10^{-2}$
3.
125
1. $5 \cdot 3$
1. $20 \times 10^{-15}$
2. $85 \times 10^{-3}$
4.
$1351.9 .31 .72 \times 10^{-7}$
1. $2010^{-3}$ 


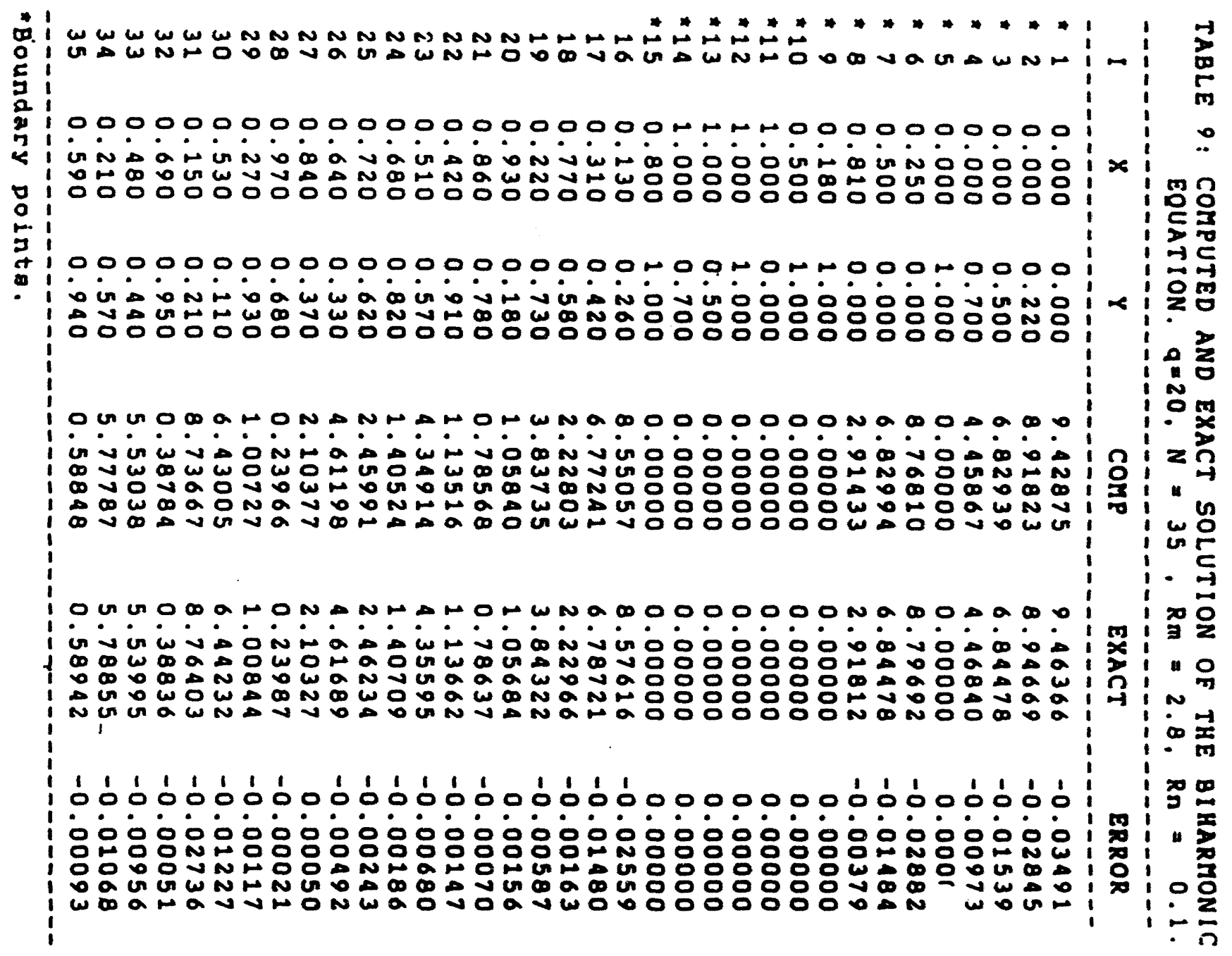


TABLE 10: COMPUTED AND EXACT SOLUTION OF BIHARMONIC EQUATION. $q=1, N=25, R m=1.5, R n=0.3$.

\begin{tabular}{|c|c|c|c|c|c|}
\hline 1 & $x$ & $Y$ & COMP & EXACT & ERROR \\
\hline 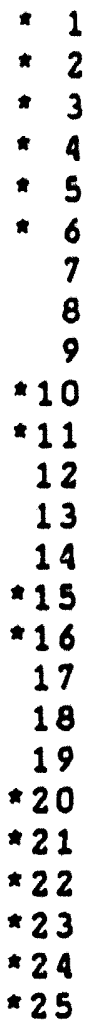 & $\begin{array}{l}0.000 \\
0.250 \\
0.500 \\
0.750 \\
1.000 \\
0.000 \\
0.250 \\
0.500 \\
0.750 \\
1.000 \\
0.000 \\
0.250 \\
0.500 \\
0.750 \\
1.000 \\
0.000 \\
0.250 \\
0.500 \\
0.750 \\
1.000 \\
0.000 \\
0.250 \\
0.500 \\
0.750 \\
1.000\end{array}$ & $\begin{array}{l}0.000 \\
0.000 \\
0.000 \\
0.000 \\
0.000 \\
0.250 \\
0.250 \\
0.250 \\
0.250 \\
0.250 \\
0.500 \\
0.500 \\
0.500 \\
0.500 \\
0.500 \\
0.750 \\
0.750 \\
0.750 \\
0.750 \\
0.750 \\
1.000 \\
1.000 \\
1.000 \\
1.000 \\
1.000\end{array}$ & $\begin{array}{l}0.47075 \\
0.43843 \\
0.34288 \\
0.19193 \\
0.00000 \\
0.43843 \\
0.40835 \\
0.31876 \\
0.17707 \\
0.00000 \\
0.34288 \\
0.31876 \\
0.24853 \\
0.13783 \\
0.00000 \\
0.19193 \\
0.17707 \\
0.13783 \\
0.07627 \\
0.00000 \\
0.00000 \\
0.00000 \\
0.00000 \\
0.00000 \\
0.00000\end{array}$ & $\begin{array}{l}0.47318 \\
0.43985 \\
0.34224 \\
0.18907 \\
0.00000 \\
0.43985 \\
0.40895 \\
0.31839 \\
0.17606 \\
0.00000 \\
0.34224 \\
0.31839 \\
0.24836 \\
0.13772 \\
0.00000 \\
0.18907 \\
0.17606 \\
0.13772 \\
0.07675 \\
0.00000 \\
0.00000 \\
0.00000 \\
0.00000 \\
0.00000 \\
0.00000\end{array}$ & $\begin{array}{r}-0.00243 \\
-0.00142 \\
0.00064 \\
0.00285 \\
0.00000 \\
-0.00142 \\
-0.00060 \\
0.00037 \\
0.00101 \\
0.00000 \\
0.00064 \\
0.00037 \\
0.00018 \\
0.00011 \\
0.00000 \\
0.00285 \\
0.00101 \\
0.00011 \\
-0.00048 \\
.0 .00000 \\
0.00000 \\
0.00000 \\
0.00000 \\
0.00000 \\
0.00000\end{array}$ \\
\hline
\end{tabular}

* Boundary pointo. 


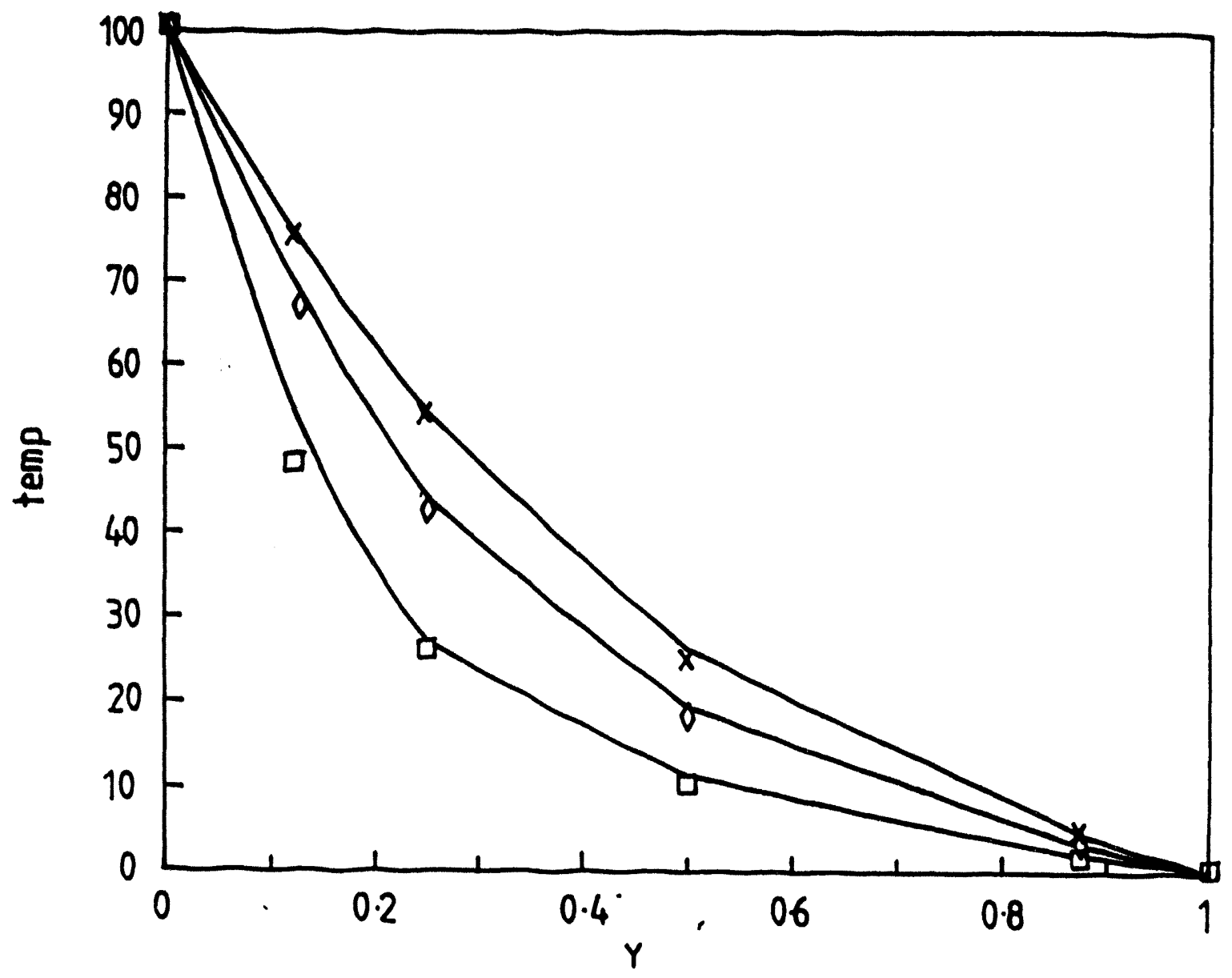

Fig1.Variation of temperature along the $Y$ - axis for different values of $X$. The contnuous lines from the top to bottom denote the $M Q$ solution for $X=0.5,0.25$ and 0.125 respectively. The symbols denote the corresponding exact solution. $N=42, R m=0.14, R_{n}=10, E=1.06$ 


$$
w
$$

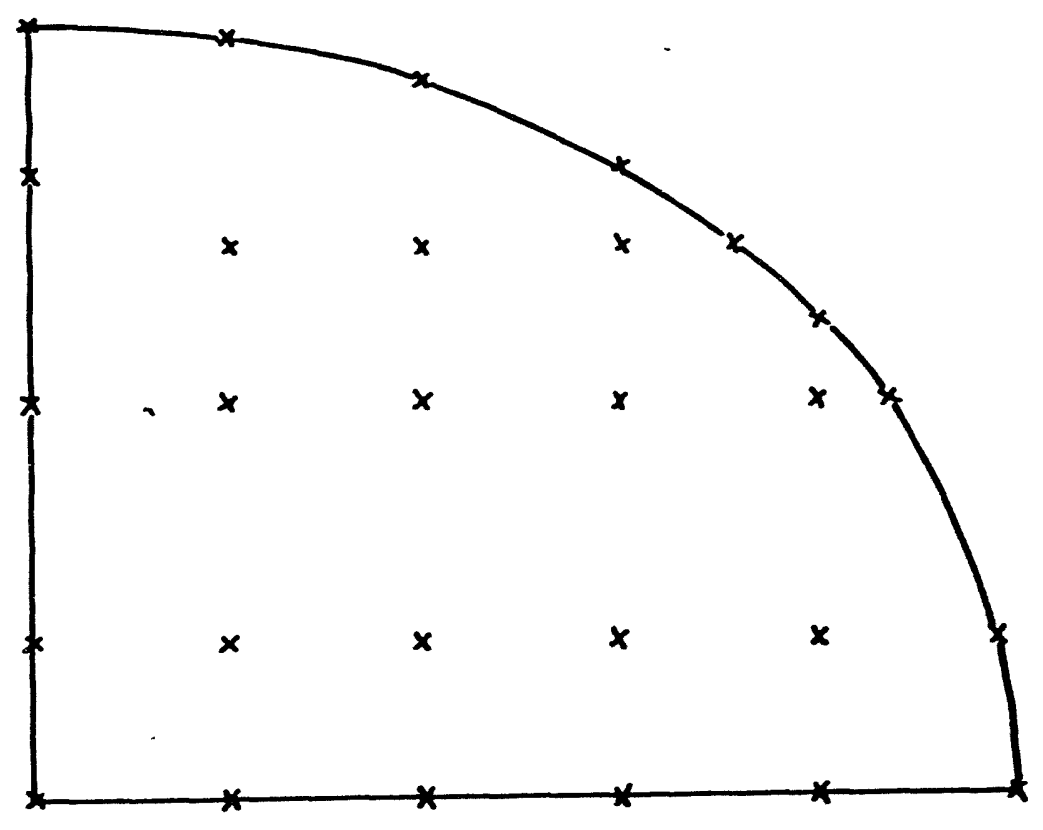

Fig. 2. Location of grid points in an ellipse. 


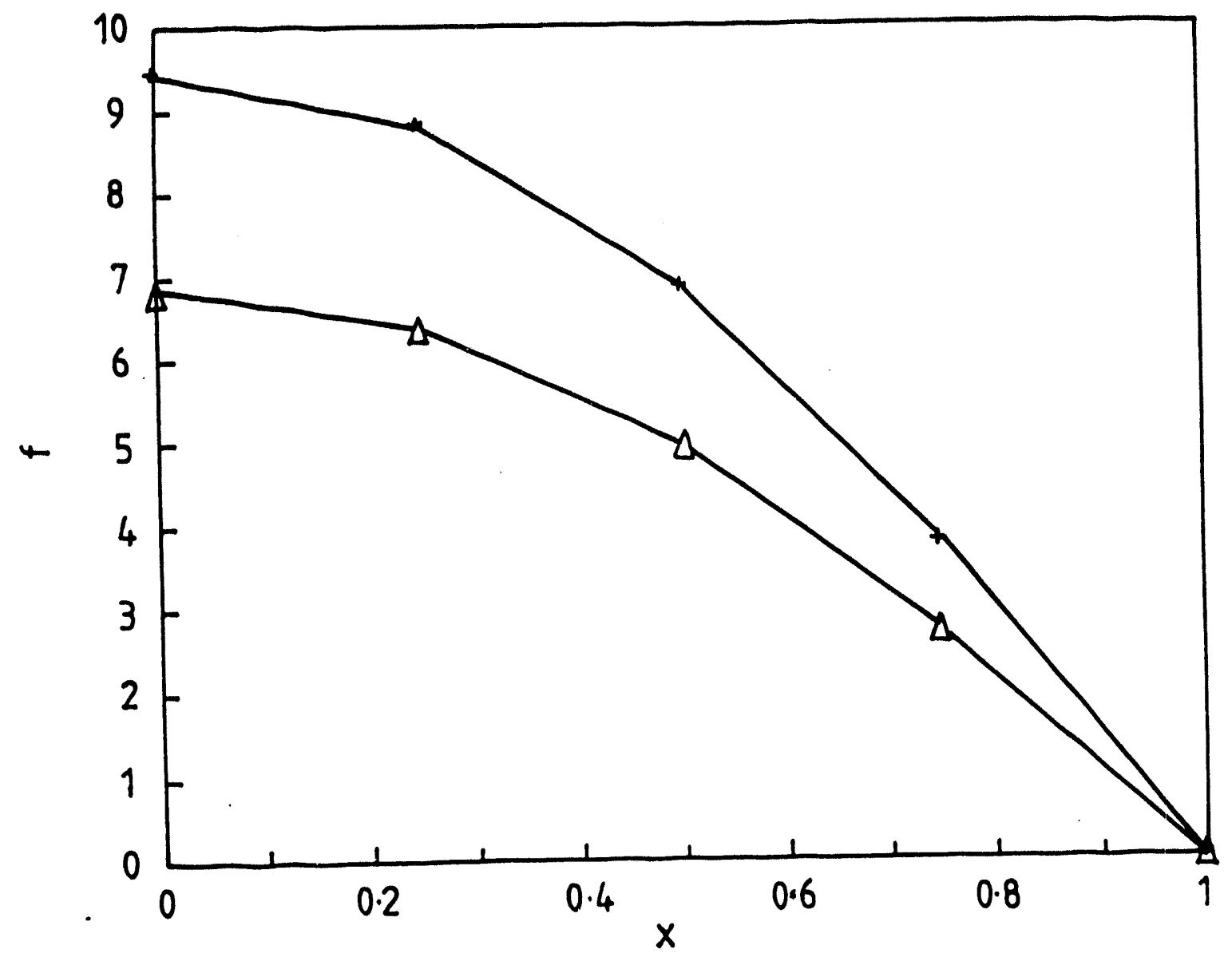

Fig.3. Computed (continuous line) and exact (symbols) solution of the biharmonic equation versus $X$ for $Y=0$ and $Y=0.5$. $+Y=0$; $\Delta Y=0.5$, Here $q=20, N=25, R m=2.2, R n=0.15, E=3.89 \times 10^{-4}$. 

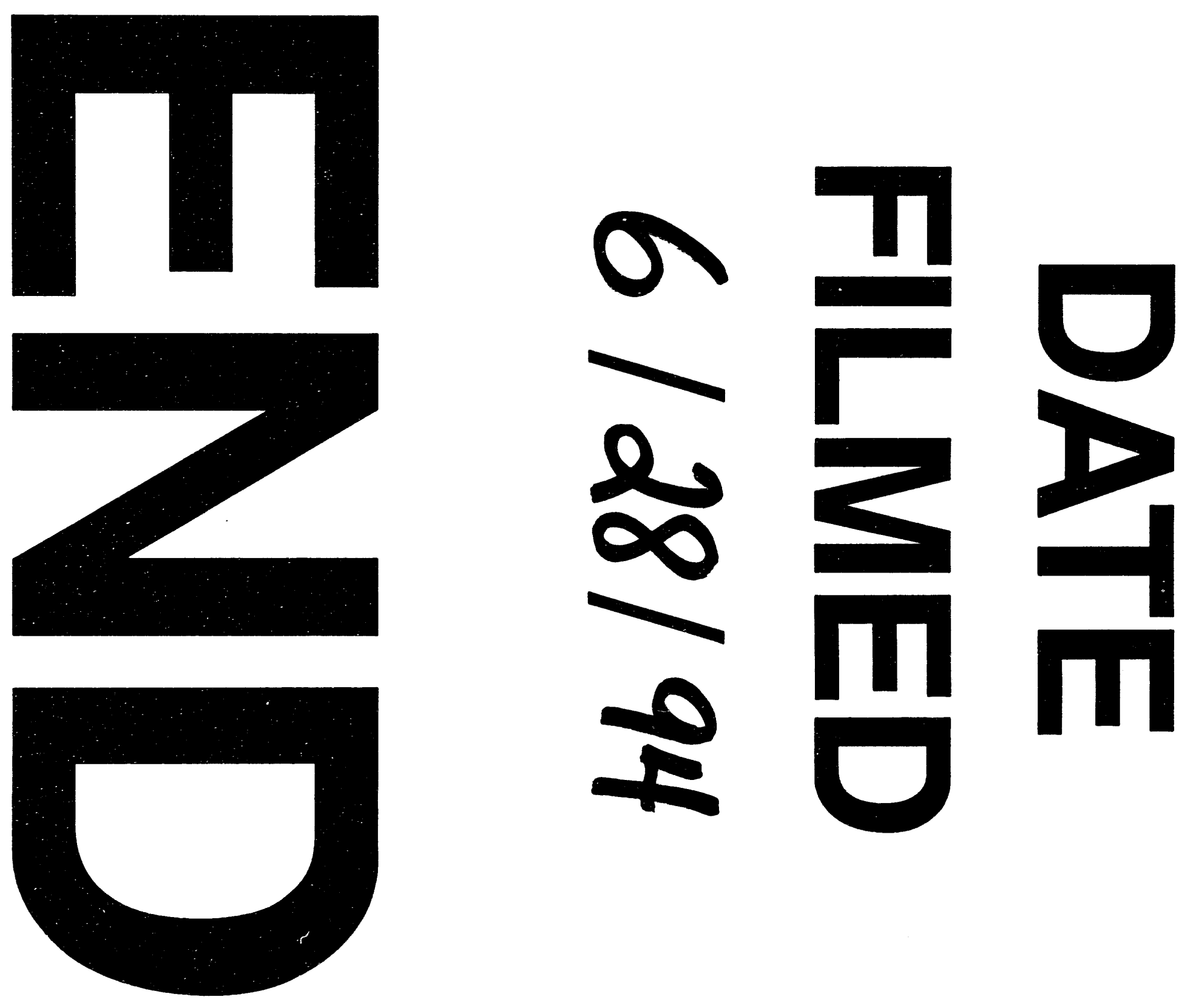
